\title{
Oxidative Stress and Inflammatory Response in Early Stage of Diffuse Axonal Injury in Rats
}

\author{
Xudong Ma, Jinning Song*, Yonglin Zhao \\ Department of Neurosurgery, The First Affiliated Hospital, Medical School of Xi'an Jiaotong University, Xi'an, \\ China \\ Email: ’Jinnings@126.com
}

Received 9 September 2015; accepted 6 October 2015; published 9 October 2015

Copyright $@ 2015$ by authors and Scientific Research Publishing Inc.

This work is licensed under the Creative Commons Attribution International License (CC BY). http://creativecommons.org/licenses/by/4.0/

(c) (i) Open Access

\begin{abstract}
Objective: To investigate the role of oxidative stress and immunoinflammatory reaction in early stage of diffuse axonal injury (DAI). Methods: 96 adult male SD rats were divided into 2 groups ( $n$ $=48$ in each): sham group and DAI group. Rat diffuse axonal injury was induced by a rat instant lateral head rotation device, which was developed to let the rat head spin 90 degree at the moment to cause shearing injury. The modified neurological severity score (mNSS), histomorphology, PI staining, GFAP immunofluorescent staining, SOD activity, CAT activity, MDA content and western blotting (IL-6,IL-1, JNK and p-JNK) in parietal cortex were investigated at $6 \mathrm{~h}, 1 \mathrm{~d}$ and $3 \mathrm{~d}$ after DAI. Results: The neurological severity scores, GFAP positive cell, PI positive cells, MDA, IL-6, IL-1, JNK and p-JNK were significantly increased and the SOD and CAT activities were decreased after DAI. Conclusion: Oxidative stress and immunoinflammatory reaction played important roles in DAI pathophysiological process in acute phase.
\end{abstract}

\section{Keywords}

Diffuse Axonal Injury (DAI), Oxidative Stress, Immunoinflammatory Reaction

\section{Introduction}

Diffuse axonal injury (DAI) is a special pathological pattern of traumatic brain injury (TBI), which is characterized with axonotmesis and axonal balls formation and associated with poor outcomes [1]-[3]. TBI is not a single pathophysiological event but a complex disease process including primary and secondary injury mechanisms. The primary injury is the result of the immediate mechanical disruption of brain tissue that occurs at the time of

\footnotetext{
"Corresponding author.
}

How to cite this paper: Ma, X.D., Song, J.N. and Zhao, Y.L. (2015) Oxidative Stress and Inflammatory Response in Early Stage of Diffuse Axonal Injury in Rats. Journal of Biosciences and Medicines, 3, 9-16. 
exposure to the external force and includes contusion, damage to blood vessels (hemorrhage) and axonal shearing, in which the axons of neurons are stretched and torn [4] [5]. Secondary injury evolves after the primary injury and is the result of cascades of metabolic, cellular and molecular events including: glutamate excitotoxicity, perturbation of cellular calcium homeostasis, increased free radical generation and lipid peroxidation, mitochondrial dysfunction, inflammation and apoptosis [6] [7]. Studies have shown that excessive production of reactive oxygen species (ROS) and inflammatory factors play an important role in the pathogenesis of secondary brain injury after TBI [8]-[10]. As a special pathological pattern of TBI, the pathogenesis of DAI may be also associated with oxidative stress and immunoinflammatory reaction, which need to be investigated.

\section{Materials and Methods}

\subsection{Instant Rotation Injury Model of DAI}

All specific pathogen-free male Sprague-Dawley rats were supplied by the Experimental Animal Center at Xi'an Jiaotong University in China. All experimental protocols conformed to the guidelines of the National Institutes of Health [11]. A total of 96 rats were randomly divided into 2 groups: (1) DAI group (DAI; $n=48$ ) and (2) Sham group (Sham; $\mathrm{n}=48$ ). All of the two groups were further divided into 3 subgroups, including $6 \mathrm{~h}, 1 \mathrm{~d}$ and $3 \mathrm{~d}$ after injury ( $\mathrm{n}=16$ per subgroup).

Rats were anesthetized by intra-peritoneal injection with $100 \mathrm{~g} / \mathrm{L}$ chloral hydrate solution $(2 \mathrm{~mL} / \mathrm{kg})$. The heads of rats were fixed to the instant lateral head rotation device and rotated immediately in the coronal plane by $90^{\circ}$ to the anticlockwise direction to make DAI models [12]. After injury, the rats were transferred to a heating pad maintained at $37^{\circ} \mathrm{C}$. Sham-injured control rats underwent anesthesia, but did not undergo trauma.

\subsection{Neurological Deficit Assessment}

In all animals, modified neurological severity score(mNSS) test was performed to assess neurological deficits before injury and at $1 \mathrm{~h}, 6 \mathrm{~h}, 1 \mathrm{~d}$ and $3 \mathrm{~d}$ after injury. Neurological function was graded on a scale of 0 to 18 (normal score, 0; maximal deficit score, 18). The mNSS is a composite of motor, sensory, reflex, and balance tests [13]. In the severity scores of injury, 1 score point is awarded for the inability to perform the test or for the lack of a tested reflex; thus, the higher score, the more severe is the injury.

\subsection{Histology}

For histology, animals were anesthetized with $100 \mathrm{~g} / \mathrm{L}$ chloral hydrate solution ( $3 \mathrm{~mL} / \mathrm{kg}$, intraperitoneally) and transcardially perfuse with heparinized phosphate-buffered saline, followed by $40 \mathrm{~g} / \mathrm{L}$ paraformaldehyde solution. The brains were fast removed and refixed by immersion in $40 \mathrm{~g} / \mathrm{L}$ paraformaldehyde for $24 \mathrm{~h}$. The samples were progressively dehydrated with ethanol and dimethylbenzene and then embedded with paraffin. Brain coronal serial sections through the hippocampus were prepared for hematoxylin-eosin (HE) staining $(4 \mu \mathrm{m})$, Nissl staining $(4 \mu \mathrm{m})$, and silver impregnation $(12 \mu \mathrm{m})$ for histopathology examination.

\subsection{Propidium Iodide (PI) Staining and GFAP Immunofluorescence Staining}

Whole brains were removed immediately from the calvaria after anesthetization and phosphate-buffered saline perfusion. Tissue slabs were frozen immediately on the surface of the liquid nitrogen and cut at $12 \mu \mathrm{m}$ thickness every $200 \mu \mathrm{m}$ interval on a freezing microtome. For PI staining, the sections were mounted on subbed slides and coverslipped using fluorescent mounting medium. For GFAP immunofluorescent staining, all sections were first blocked with $10 \%$ normal goat serum blocking solution from the same species as the secondary antibody, containing $50 \mathrm{~g} / \mathrm{L}$ BSA and $0.1 \%$ Triton X-100, for $2 \mathrm{~h}$ at room temperature to avoid nonspecific staining. Then the sections were incubated with primary antibody for anti-GFAP (Cell Signaling Technology, USA) overnight at $4^{\circ} \mathrm{C}$, which was followed by the TITC-conjugated secondary antibody for $2 \mathrm{~h}$ at $37^{\circ} \mathrm{C}$ and DAPI for 5 minutes at room temperature. 3 sections of each brain were observed to count PI-positive cells or GFAP-positive cells by 3 random visual field $(\times 400)$ at cortico-medullary border area with fluorescence microscope.

\subsection{Measurement of Antioxidant Enzymatic Activity and Oxidative Product Level}

Rats were euthanized under anesthesia, and then perfuse through the ascending aorta artery with $200 \mathrm{ml}$ chilled 
PBS (0.1 mol/L, pH 7.4). The bilateral cortical tissues were dissected and homogenized in chilled PBS (0.1 $\mathrm{mol} / \mathrm{L}$, $\mathrm{pH} 7.4$ ), and then centrifuged at $10,000 \mathrm{r} / \mathrm{min}$ at $4^{\circ} \mathrm{C}$ for $10 \mathrm{~min}$. The supernatants were collected, aliquoted, and stored at $-80^{\circ} \mathrm{C}$ until analysis. The activities of SOD and CAT and the level of MDA were measured using commercial kits (A001, A007, A003; Nanjing Jiancheng Bioengineering Institute, China). All standards and samples were conducted in duplicates and all assays were run in triplicates. The tissue protein concentration was assayed using the BCA protein assay reagent.

\subsection{Western Blotting}

The expression of IL-6, IL-1 $\beta$, p-JNK and JNK in cortex were determined by western blotting. At pre-determined time points, animals were anesthetized and perfuse with $200 \mathrm{ml}$ chilled PBS (0.1 mol/L, pH 7.4). The bilateral cortex of the brain were removed immediately and stored in liquid nitrogen until the following analysis. Frozen tissues were homogenized and lysed with RIPA lysis buffer. Protein concentration was detected using the BCA protein assay reagent. Samples were resolved by electrophoresis ( $120 \mathrm{~V}, 40 \mathrm{~min})$ on a $12 \%$ SDSPAGE gel. Then the proteins were transferred onto a PVDF membrane and the nonspecific binding was blocked with $50 \mathrm{~g} / \mathrm{L}$ non-fat milk at room temperature for $1 \mathrm{~h}$. This was followed by incubation with rabbit polyclonal anti-IL-6 (1:200), rabbit polyclonal anti-IL-1 $\beta$ (1:200), rabbit monoclonal anti-JNK (1:1000) or rabbit monoclonal anti-p-JNK (1:1000) (Cell Signaling Technology, USA) overnight at $4^{\circ} \mathrm{C}$. Afterwards, HRP-anti-rabbit IgG (Santa Cruz Biotechnology, USA) was applied to probe these specific antibodies. Subsequently, these specific antibodies were detected by using ECL Western blotting detection (Millipore, USA) and the fluorescence excitation was imaged on X-ray film. Normalization was based on the protein level of $\beta$-actin and analyzed by Quantity One 4.62 software.

\subsection{Statistical Analysis}

All statistical analysis was performed using SPSS 13.0 (SPSS Inc. USA). All data were presented as mean \pm SEM. All data were analyzed by analysis of t-test when normality and homogeneity of variance assumptions are satisfied. Significance was set at $P<0.05$.

\section{Result}

\subsection{Neurological Deficit Assessment after DAI}

The animals in DAI group became comatose immediately after strike, which last 10 - 30 minutes, and then experienced neurologic impairment including motor dysfunction, sensory disturbance, balance function disorder, reflexes absent and abnormal movements. The animals showed significantly higher mNSS mean score, on half of the more serious neurological deficit, at $1 \mathrm{~h}, 6 \mathrm{~h}, 1 \mathrm{~d}$ and $3 \mathrm{~d}$ compared with the sham-injured group $(P<0.01)$ (Figure 1).

\subsection{Morphology Changes in Prefrontal Cortex Tissue after DAI}

The morphological changes of HE staining (Figure 2(A)), Nissl staining (Figure 2(B)) and Glees silver staining (Figure 2(C)) in prefrontal cortex after DAI are shown in Figure 2. Normal morphological features in prefrontal cortex were observed in the sham group (Figures 2(A)-(C) Sham), where the cortical structure was intact, cells line up serially with a round or oval clear borderline, the cellular plasma were plenty and bright and the nucleolus were clear and centered, under HE staining and Nissl staining. Neuronal axons were smooth, slender and continuous under silver staining. However, in the DAI group, part of cortical neurons were obviously shrunken and pyknotic and some of them also displayed irregular shape and a tangled appearance (Figure 2(A) and Figure 2(B) DAI). Silver staining showed swelled axons and axonal balls after injury (Figure 2(C) DAI). These morphological changes arose $6 \mathrm{~h}$ after DAI, and were most obvious at $3 \mathrm{~d}$ after DAI.

\subsection{PI-Positive Cells Increased after DAI}

Late apoptotic cells and necrotic cells were marked by PI staining with red fluorescence (Figure 3(A)). In DAI group, PI-positive cell count increased from $43.1 \pm 7.3 /$ view to $149.4 \pm 14.7 /$ view at 6 h, $44.2 \pm 9.0 /$ view to 


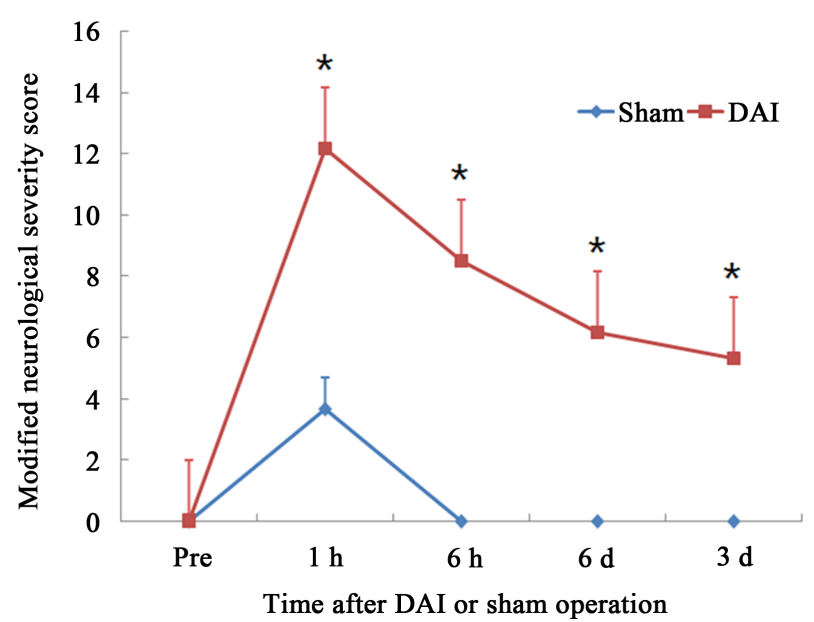

Figure 1. Changes of modified neurological severity score in DAI rats. The animals in DAI group showed significantly higher mNSS mean score, on half of the more serious neurological deficit, at $1 \mathrm{~h}, 6 \mathrm{~h}, 1 \mathrm{~d}$ and $3 \mathrm{~d}$ compared with the sham-injured group $\left({ }^{*} P<0.01\right)$.

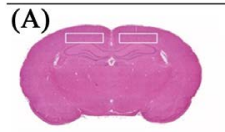

(B)

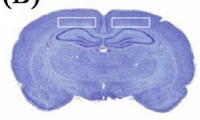

(C)

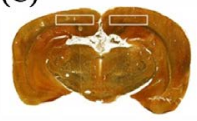

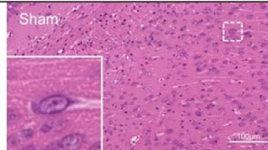
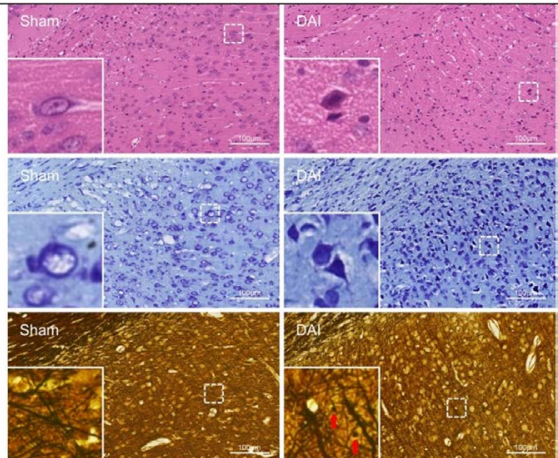

Figure 2. Morphology changes in prefrontal cortex tissue 3d after DAI. (A) HE staining. Normal morphological features were observed in sham group. In the DAI group, parts of cortical neurons were obviously shrunken and pyknotic and some of them also displayed irregular shape and a tangled appearance; (B) Nissl staining. Normal morphological features were observed in sham group. In the DAI group, neurons were pyknotic with irregular shape, the cellular plasma and nucleolus disappeared, and the Nissl substance lost or dissolved; (C) Glees silver staining. Neuronal axons were smooth, slender and continuous in sham group, while swelled axons and axonal balls (red arrows) could be observed after DAI.

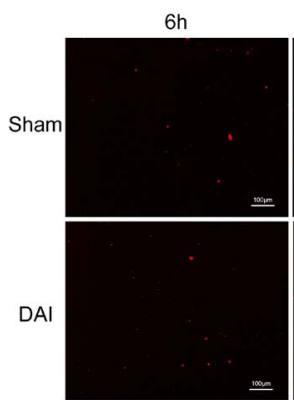

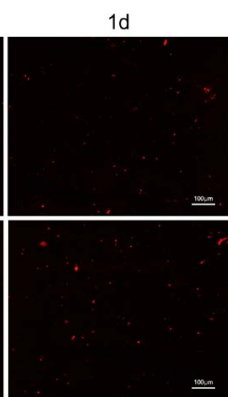

(A)

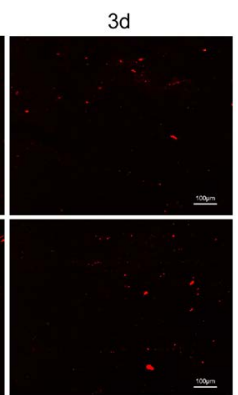

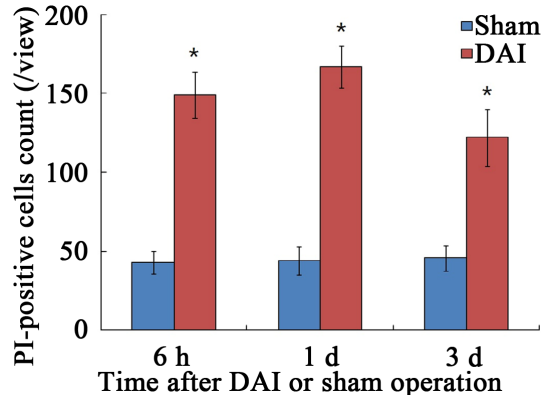

(B)

Figure 3. PI staining and count of PI-positive cells in prefrontal cortex tissue after DAI. (A) PI staining. Late apoptotic cells and necrotic cells were stained by PI with red fluorescence in each group at $6 \mathrm{~h}, 1 \mathrm{~d}$ and $3 \mathrm{~d}$; (B) PI-positive cell count. In DAI group, PI-positive cell count significantly increased at $6 \mathrm{~h}, 1 \mathrm{~d}$, and $3 \mathrm{~d}$, compared with sham group $\left({ }^{*} P<0.05\right)$. 
$167.2 \pm 13.5 /$ view at $1 \mathrm{~d}$ and $46.2 \pm 8.0 /$ view to $141.2 \pm 18.1 /$ view at $3 \mathrm{~d}$, compared with sham group. The changes of PI-positive cell count among each groups were statistically significance by t-test method $(P<0.01)$ (Figure 3(B)).

\subsection{Activation of Astrocytes after DAI}

The astrocytes were immunostained with astrocytes marker GFAP antibody and the cell nucleus were stained with DAPI in sham group and DAI group. The colocalizations of GFAP and DAPI as GFAP-positive cells were counted (Figure 4(A)). In DAI group, the count of GFAP-positive cells increased from $4.8 \pm 0.9$ /view to $5.2 \pm$ 1.5, $6.3 \pm 2.0$ and $10.3 \pm 1.5$ /view at 6 h, $1 \mathrm{~d}$ and $3 \mathrm{~d}$ (Figure 4(B)).

\subsection{Explosion of Oxidative Stress after DAI}

The activities of SOD and CAT in cortex were detected at $6 \mathrm{~h}, 1 \mathrm{~d}$ and $3 \mathrm{~d}$ in sham group and DAI group. The result showed that the activity of SOD in cortex was significantly decreased from $157.7 \pm 13.9 \mathrm{U} / \mathrm{mgprot}$ and $145.5 \pm 10.2 \mathrm{U} / \mathrm{mgprot}$ to $97.8 \pm 13.8 \mathrm{U} / \mathrm{mgprot}$ and $81.0 \pm 15.1 \mathrm{U} / \mathrm{mgprot}$ in $1 \mathrm{~d}$ and $3 \mathrm{~d}$ after DAI $(P<0.05$, Figure 5(A)). The activity of CAT was also significantly decreased from $15.5 \pm 2.0$ U/gprot and $15.1 \pm 1.5$ U/gprot to $8.7 \pm 1.2 \mathrm{U} /$ gprot and $8.0 \pm 1.5 \mathrm{U} /$ gprot in $1 \mathrm{~d}$ and $3 \mathrm{~d}(P<0.05$, Figure 5(B)). The level of oxidative product MDA in cortex was also measured at $6 \mathrm{~h}, 1 \mathrm{~d}$ and $3 \mathrm{~d}$ in each group. The level of MDA was significantly elevated from $4.6 \pm 0.9 \mathrm{nmol} / \mathrm{mgprot}$ and $4.7 \pm 1.0 \mathrm{nmol} / \mathrm{mgprot}$ to $11.4 \pm 1.1 \mathrm{nmol} / \mathrm{mgprot}$ and $15.7 \pm 1.5$ $\mathrm{nmol} / \mathrm{mgprot}$ in $1 \mathrm{~d}$ and 3d after DAI $(P<0.05$, Figure 5(C)).

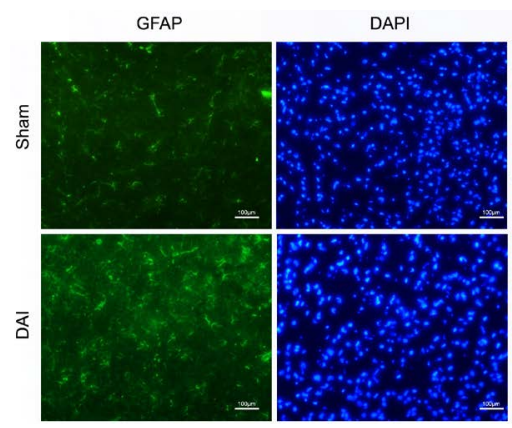

(A)

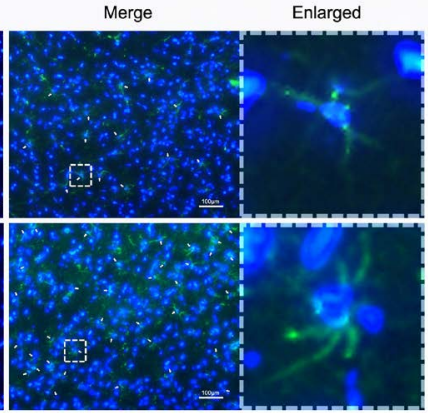

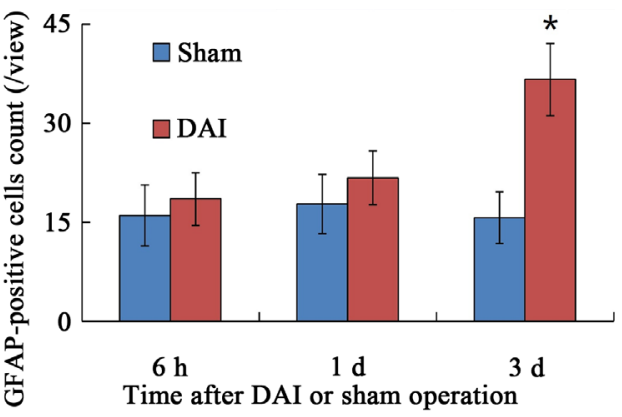

(B)

Figure 4. GFAP immunofluorescent staining and count of GFAP-positive cells in prefrontal cortex tissue after DAI. (A) GFAP immunofluorescent staining. The cryosections from each group were immunostained with astrocytes marker GFAP antibody and the cell nucleus was stained with DAPI. The colocalizations of GFAP and DAPI as GFAP-positive cells were shown in merged images; (B) PI-positive cell count. GFAP-positive cells at 3 d significantly rose after DAI. ${ }^{*} P<0.05$ vs. sham group.

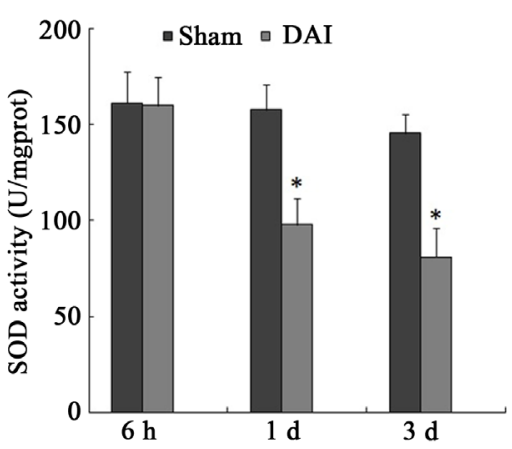

(A)

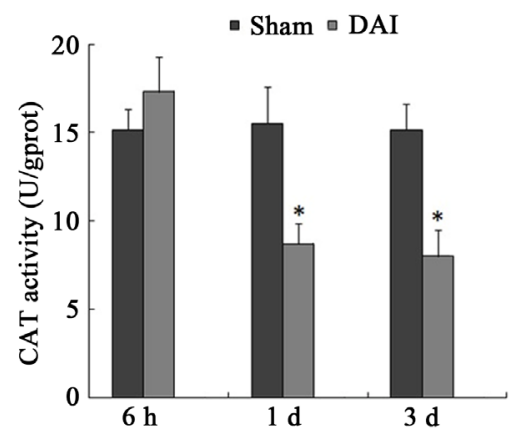

(B)

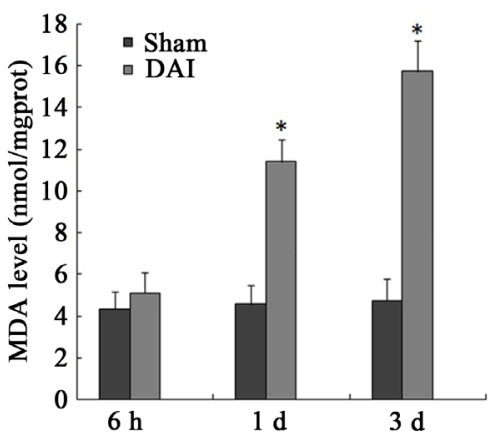

(C)

Figure 5. Oxidative stress changes after DAI. (A), (B) The activities of SOD and CAT in cortex were significantly decreased from $1 \mathrm{~d}$ to $3 \mathrm{~d}$ after DAI. (C) The level of MDA was significantly elevated from $1 \mathrm{~d}$ to $3 \mathrm{~d}$ after DAI. ${ }^{*} P<0.05$ vs. sham group. 


\subsection{Inflammatory Factors Substantially Produced after DAI}

The expression of IL-1 $\beta$, IL-6, JNK and p-JNK in cortex were detected by western blotting at $6 \mathrm{~h}, 1 \mathrm{~d}$ and $3 \mathrm{~d}$ in each group. The results showed that the IL-1 $\beta$, IL-6 and p-JNK expressions and the ratio of p-JNK to JNK were increased and peaked at $1 \mathrm{~d}$ after DAI (Figure 6).

\section{Discussion}

As one type of primary brain injury, DAI has specific traumatic mechanisms. DAI is not only related to the degree of violence proportionally, but is associated with the nature and mode of action of violence. The shear force caused by rotational acceleration is the main reason for DAI, which often happens with instant lateral head rotation [14] [15]. At present, the methods of setting up animal models mainly conclude: fluid percussion injury (FPI), controlled cortical impact (CCI) injury, weight-drop impact acceleration injury and instantaneous rotation injury (IRI) [4] [14]. As the instantaneous rotation injury model could well simulate the mechanical mechanism of DAI, we used the rat instant lateral head rotation device developed by Liu [12] to establish rat DAI model. By observing axons in frontal junction of the cortex using Gless silver staining, we identified the flexion and bead-like changes of axons and the characteristic formation of axonal retraction balls as specific changes associated with DAI. mNSS also showed remarkable neurological impairment after modeling, and demonstrated severe brain injury. Therefore, both the pathological findings and the mNSS demonstrated that the DAI model was successfully established.

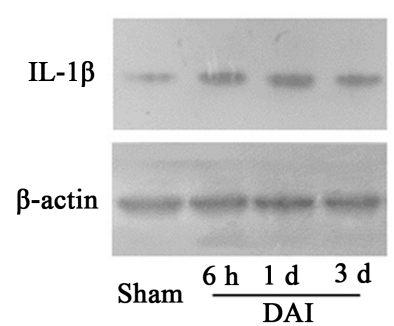

(A)

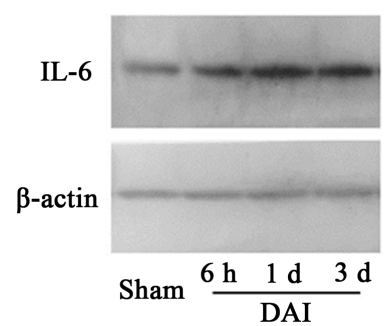

(C)

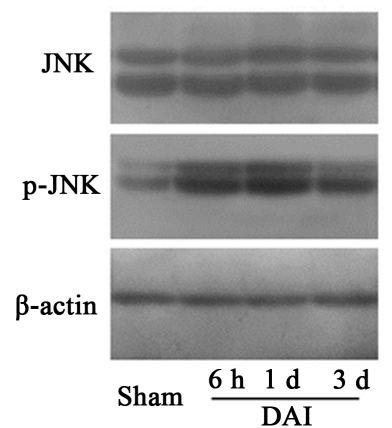

(E)

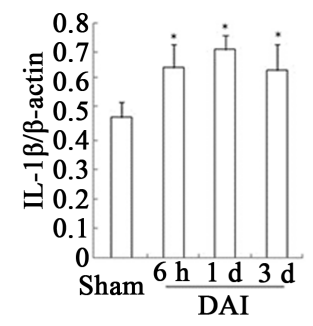

(B)

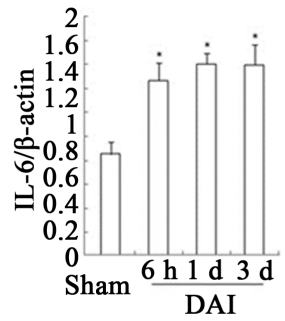

(D)

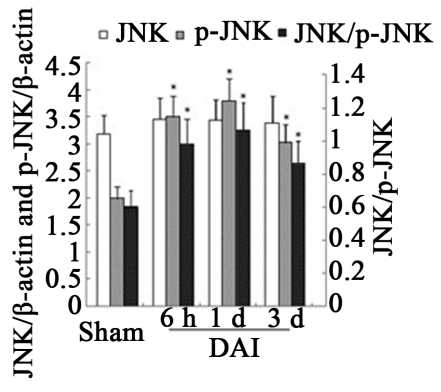

(F)

Figure 6. Changes of inflammatory factors after DAI. (A), (C), (E) Western blotting analysis of IL-1 $\beta$, IL-6, JNK and p-JNK at 6 h, $1 \mathrm{~d}$ and $3 \mathrm{~d}$ in sham group and DAI group. (B), (D), (F) The IL-1 $\beta$, IL-6 and p-JNK expressions and the ratio of p-JNK to JNK were increased and peaked at $1 \mathrm{~d}$ after DAI. ${ }^{*} P<0.05$ vs. sham group. 
DAI is not a single pathophysiological event but a complex disease process, and it causes structural damage and functional deficits that are due to both primary and secondary injury mechanisms [4]. As the primary injury occurs immediately after the moment of trauma, it can only be preventable (for example, through the use of a seat belt or helmet). By contrast, the elongated nature of secondary injury development provides a window of opportunity for therapeutic intervention, which may prevent and/or reduce secondary brain damage [15] [16]. Secondary injury evolves after the primary injury and is the result of cascades of metabolic, cellular and molecular events including: glutamate excitotoxicity, perturbation of cellular calcium homeostasis, increased free radical generation and lipid peroxidation, mitochondrial dysfunction, inflammation and apoptosis [6] [7]. It has been proved that oxidative stress and inflammatory response promote and influence each other, which lead to cascade reaction and exacerbate secondary injury in FPI or CCI models [9] [17]. Inflammatory responses following neural injury have been suggested as an important mechanism in several types of neural system disease. Well-investigated cytokines include the IL- 1 family, IL-6, IL-10, and TNF- $\alpha$, which likely play an important role in inflammatory reactions following TBI. It is well-known that the IL-1 family has extensive inflammatory functions such as stimulating T-cells and macrophages as well as leading to the secretion of other cytokines and chemokines. In addition, Campbell et al. [18] also reported that the overexpression of IL-1 $\beta$ has a strong relation with axonal injury. IL-6 is an important inflammatory cytokine and has a wide range of effects. It affects both pro-inflammatory and anti-inflammatory responses via regulation of the activity of immunocytes such as neutrophils, lymphocytes, and NK-cells as well as the induction of the release of soluble TNFR and IL-1 receptor antagonists [19] [20]. JNK signaling is the shared pathway linking neuroinflammation, blood-brain barrier disruption, and oligodendroglial apoptosis in the white matter injury of the immature brain [21].

Therefore, we investigated the changes of radical generation and inflammatory cytokines in DAI models. The result showed that the activities of antioxidant enzymes significantly decreased and the level of lipid peroxidation increased after DAI, which indicated that excessive production of ROS and reduction of antioxidant defense systems played an important role in the pathogenesis of DAI. In addition, the pro-inflammatory cytokines IL-6 and IL-1 $\beta$ and inflammatory related pathway p-JNK were elevated after DAI, which indicated that inflammatory response participated in the secondary injury mechanisms of DAI. The immunoinflammatory reaction induced by astrocytes play an important role in the mechanisms of DAI. The injury stimulates hypertrophic and proliferative changes in astrocytes and induces it produce inflammatory factors involved in the initiation of immunologic cascade [8]. Our results showed that astrocytes got hypertrophy and proliferation after DAI, which also indicated that immunoinflammatory reaction played an important role in the secondary injury mechanisms of DAI.

\section{Conclusion}

In conclusion, we found that IRI-induced DAI models showed significant brain injuries characterized by neurological dysfunction, characteristic formation of axonal retraction balls and neuron necrosis and apoptosis after DAI. In addition, our results showed that oxidative stress and immunoinflammatory reaction broke out after DAI in early stage, which played important roles in pathological process of DAI.

\section{References}

[1] Romodanovsky, P.O. (2013) Certain Aspects of Diffuse Axonal Injury to the Brain in the Case of Head Trauma. Sudebno-meditsinskaya Ekspertiza, 56, 18-20.

[2] Gennarelli, T.A., Thibault, L.E. and Graham, D.I. (1998) Diffuse Axonal Injury: An Important Form of Traumatic Brain Damage. Neuroscientist, 4, 202-215. http://dx.doi.org/10.1177/107385849800400316

[3] Smith, D.H., Meaney, D.F. and Shull, W.H. (2003) Diffuse Axonal Injury in Head Trauma. Journal of Head Trauma Rehabilitation, 18, 307-316. http://dx.doi.org/10.1097/00001199-200307000-00003

[4] Xiong, Y., Mahmood, A. and Chopp, M. (2013) Animal Models of Traumatic Brain Injury. Nature Reviews Neuroscience, 14, 128-142. http://dx.doi.org/10.1038/nrn3407

[5] Masel, B.E. and Dewitt, D.S. (2010) Traumatic Brain Injury: A Disease Process, Not an Event. Journal of Neurotrau$m a, 27,1529-1540$. http://dx.doi.org/10.1089/neu.2010.1358

[6] Song, J.N. and Li, Y. (2014) Research Advances of Secondary Brain Injury Caused by Diffuse Axonal Injury and Its Therapeutic Strategies. Journal of Xi' an Jiaotong University, Medical Sciences, 35, 717-725.

[7] Gaetz, M. (2004) The Neurophysiology of Brain Injury. Clinical Neurophysiology, 115, 4-18. http://dx.doi.org/10.1016/S1388-2457(03)00258-X 
[8] Laird, M.D., Vender, J.R. and Dhandapani, K.M. (2008) Opposing Roles for Reactive Astrocytes Following Traumatic Brain Injury. Neurosignals, 16, 154-164. http://dx.doi.org/10.1159/000111560

[9] Rodriguez-Rodriguez, A., Egea-Guerrero, J.J., Murillo-Cabezas, F. and Carrillo-Vico, A. (2014) Oxidative Stress in Traumatic Brain Injury. Current Medicinal Chemistry, 21, 1201-1211. http://dx.doi.org/10.2174/0929867321666131217153310

[10] Mustafa, A.G. and Al-Shboul, O.A. (2013) Pathophysiology of Traumatic Brain Injury. Neurosciences, 18, $222-234$.

[11] National Research Council (1996) Guide for the Care and Use of Laboratory Animals. 7th Edition, National Academy Press, Washington DC.

[12] Li, Y., Song, J.N., Liu, X.B., Zhang, M., Sun, P., Li, D., Jin, T. and Wang, J.F. (2013) High Expression of STIM1 in the Early Stage of Diffuse Axonal Injury. Brain Research, 1495, 95-102. http://dx.doi.org/10.1016/j.brainres.2012.12.005

[13] Chen, J.L. (2001) Therapeutic Benefit of Intravenous Administration of Bone Marrow Stromal Cells after Cerebral Ischemia in Rats. Stroke, 32, 1005-1011. http://dx.doi.org/10.1161/01.STR.32.4.1005

[14] Krave, U., Hojer, S. and Hansson, H.A. (2005) Transient, Powerful Pressures Are Generated in the Brain by a Rotational Acceleration Impulse to the Head. European Journal of Neuroscience, 21, 2876-2882. http://dx.doi.org/10.1111/j.1460-9568.2005.04115.x

[15] Chen, G., Go, L. and Mao, B. (2002) Biomechanical Mechanism of Diffuse Axonal Injury. Journal of Biomedical Engineering, 19, 500-504.

[16] Smith, D.H., Hicks, R. and Povlishock, J.T. (2013) Therapy Development for Diffuse Axonal Injury. Journal of Neurotrauma, 30, 307-323. http://dx.doi.org/10.1089/neu.2012.2825

[17] Lin, Y. and Wen, L. (2013) Inflammatory Response Following Diffuse Axonal Injury. International Journal of Medical Sciences, 10, 515-521. http://dx.doi.org/10.7150/ijms.5423

[18] Campbell, S.J., Deacon, R.M., Jiang, Y., Ferrari, C., Pitossi, F.J. and Anthony, D.C. (2007) Overexpression of IL-1beta by Adenoviral-Mediated Gene Transfer in the Rat Brain Causes a Prolonged Hepatic Chemokine Response, Axonal Injury and the Suppression of Spontaneous Behaviour. Neurobiology of Disease, 27, 151-163. http://dx.doi.org/10.1016/j.nbd.2007.04.013

[19] Tilg, H., Trehu, E., Atkins, M.B., Dinarello, C.A. and Mier, J.W. (1994) Interleukin-6 (IL-6) as an Anti-Inflammatory Cytokine: Induction of Circulating IL-1 Receptor Antagonist and Soluble Tumor Necrosis Factor Receptor p55. Blood, 83, 113-118.

[20] Scheller, J., Chalaris, A., Schmidt-Arras, D. and Rose-John, S. (2011) The Pro- and Anti-Inflammatory Properties of the Cytokine Interleukin-6. Biochimica et Biophysica Acta, 1813, 878-888. http://dx.doi.org/10.1016/j.bbamcr.2011.01.034

[21] Wang, L.W., Tu, Y.F., Huang, C.C. and Ho, C.J. (2012) JNK Signaling Is the Shared Pathway Linking Neuroinflammation, Blood-Brain Barrier Disruption, and Oligodendroglial Apoptosis in the White Matter Injury of the Immature Brain. Journal of Neuroinflammation, 9, 175. http://dx.doi.org/10.1186/1742-2094-9-175 